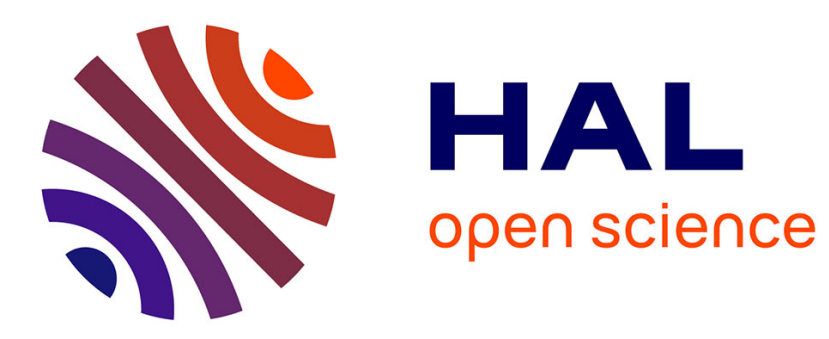

\title{
Diffraction peaks restoration and extraction in energy dispersive X-ray diffraction
}

Ferréol Soulez, Charles Crespy, Valerie Kaftandjian, Philippe Duvauchelle

\section{To cite this version:}

Ferréol Soulez, Charles Crespy, Valerie Kaftandjian, Philippe Duvauchelle. Diffraction peaks restoration and extraction in energy dispersive X-ray diffraction. Nuclear Instruments and Methods in Physics Research Section A: Accelerators, Spectrometers, Detectors and Associated Equipment, 2011, pp.10.1016/j.nima.2011.06.037. 10.1016/j.nima.2011.06.037 . hal-00605917

\section{HAL Id: hal-00605917 https://hal.science/hal-00605917}

Submitted on 4 Jul 2011

HAL is a multi-disciplinary open access archive for the deposit and dissemination of scientific research documents, whether they are published or not. The documents may come from teaching and research institutions in France or abroad, or from public or private research centers.
L'archive ouverte pluridisciplinaire HAL, est destinée au dépôt et à la diffusion de documents scientifiques de niveau recherche, publiés ou non, émanant des établissements d'enseignement et de recherche français ou étrangers, des laboratoires publics ou privés. 


\title{
Diffraction peaks restoration and extraction in energy dispersive X-ray diffraction
}

\author{
Ferréol Soulez $^{\mathrm{a}, \mathrm{b}, 1, *}$, Charles Crespy $^{\mathrm{a}}$, Valérie Kaftandjian ${ }^{\mathrm{a}}$, Philippe Duvauchelle $^{\mathrm{a}}$ \\ ${ }^{a}$ Laboratoire de Contrôle Non Destructif par Rayonnements Ionisants (CNDRI) - Insa Lyon - Universite de Lyon - 20 Avenue Albert \\ Einstein, F-69621 Villeurbanne cedex, France \\ ${ }^{b}$ Centre Commun de Quantimetrie - Université Lyon I - Universite de Lyon - 8 avenue Rockefeller, F-69373 Lyon cedex 08, France
}

\begin{abstract}
This paper proposes a method to restore energy dispersive X-ray diffraction (EDXRD) spectra and to extract diffraction peaks. It follows a maximum a posteriori approach using a physical model of the formation of the EDXRD data to remove blur caused by both the detector and the coarse angular resolution of X-ray tube based EDXRD setup. It separates peaks due to the diffraction by crystalline material from a countinuous background. Tested on real data (graphite and $\mathrm{NaCl}$ ), our algorithm acheived to detect diffraction peaks with a good precision (about $1 \mathrm{keV}$ depending on the peak position) even at high energy where very few photons were measured.
\end{abstract}

Keywords: Energy Dispersive X ray Diffraction, Non Destructive Testing, spectrum restoration, inverses problems

\section{Introduction}

X-ray diffraction (XRD) has been successfully used for many years to provide information about the crystalline structure of samples using coherent scattering of photons from an incident $\mathrm{X}$ ray beam. Powder X ray diffraction is commonly used to identify unknown substances by comparing diffraction data against a database[1, 2]. It may also be used to characterize heterogeneous solid mixtures to determine relative abundance of crystalline compounds and, when coupled with lattice refinement techniques such as Rietveld refinement, can provide structural information on unknown materials. Most diffractometers use angular dispersive $\mathrm{X}$ ray diffraction (ADXRD) in angular dispersion of a diffracted monochromatic radiation is used, as it provides more precise diffraction spectra(see [3] for a review). As detailed in ref.[3] many deconvolution and pattern fitting procedures were designed for ADXRD data.

However ADXRD presents some drawbacks as it requires most of the times a rotation of the detector and the sample. When no movement is possible in the setup (e.g. as in security screening) or when crystalline structure is dynamically changing (to monitor the change of lattice parameters with pressure or temperature), one must use Energy dispersive $\mathrm{X}$ ray diffraction (EDXRD) for which the scattering angle is fixed. In EDXRD, a collimated polychromatic beam of X-rays falls on the sample, and the spectrum of the radiation scattered at a fixed angle $\theta$ is analyzed. The spectrum thus obtained is material specific since it is linked to the atomic planar spacing $(d)$ and

\footnotetext{
* Corresponding author

Email addresses: ferreol.soulez@univ-lyon1.fr (Ferréol Soulez), valerie.kaftandjian@insa-lyon.fr (Valérie Kaftandjian)
}

to the radiation wavelength $(\lambda)$ according to Bragg law:

$$
2 d \sin \left(\frac{\theta}{2}\right)=n \lambda,
$$

where $n$ is an integer. Using an ideal diffractometer with perfect collimation and detectors, this diffraction spectrum would be composed only of lines predicted from Bragg theory.

As they provide intense radiation with known spectrum and polarization, most EDXRD experiments use synchrotron radiation $[4,5]$. Furthermore as angular aperture of synchrotron light is very small, the blurring of the spectra caused by the width of possible diffraction angles is negligible compared to the blurring effect due to the detector response[6]. As a consequence most pattern fitting algorithms[7-9] are designed for EDXRD data with synchrotron radiation and consider a spectraly invariant Gaussian peak-shape function[6, 7].

However if synchrotron is an ideal source for EDXRD, it is scarcely available and only X-ray tube can be used in laboratory or for in situ applications. Angular aperture of collimated X-ray source is quite large and consequently geometric blurring effects result in a "smoothing out" of the perfect line spectra into a less well resolved continuous spectra[10]. Very tight collimation, whilst minimizing this effect, would make inefficient use of the scattered flux available and then drastically increase the acquisition time.

As geometric effect introduces an energy variant blur, such EDXRD cannot be processed by existent algorithms developed for ADXRD or EDXRD with synchrotron source. In this paper we propose an inverse problem based method for restoration of EDXRD diffraction spectra and direct extraction of diffraction peaks from crystalline material. It uses a physical model of the measurements de- 


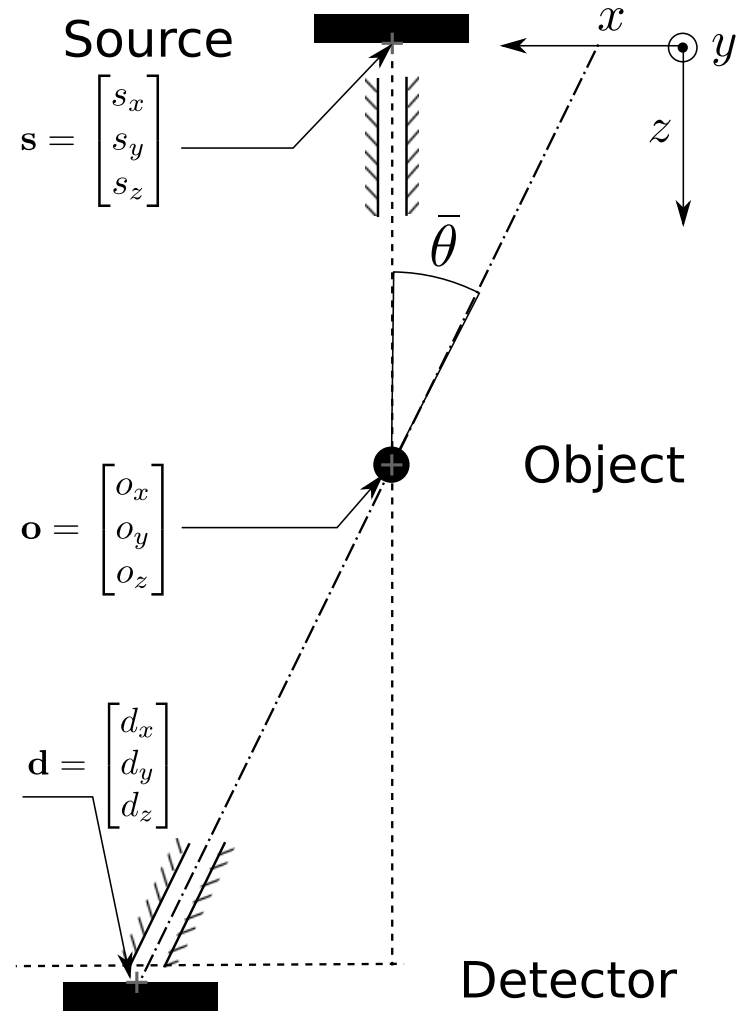

Figure 1: Schematic view of a X ray diffraction setup and notations used in the model.

scribed in section 3 . Then in section 4, following an inverse problem approach, we introduce some priors and define the maximum a posteriori (MAP) solution which is estimated (in Sec. 5) using a continuous optimization procedure. Finally, we apply our method for estimation of inter-planar spacing of real EDXRD spectrum acquired in laboratory.

\section{EDXRD setup and notation}

As depicted in figure 1, the EDXRD setup consists of a collimated poly-chromatic X-ray source and a collimated spectroscopic detector $D$ placed at nominal angle $\bar{\theta}$ of the beam axis. The studied object $O$ is at the center of the diffractometer defined as the intersection of the scatter collimator axis and the source collimator axis.

Let's suppose that we observe at angle $\bar{\theta}$ a pure crystalline powder sample under ideal conditions: using a source with flat spectrum, a diffractometer with an infinite angular resolution, a perfect detector without blur or noise. Without any other spurious effects like Compton scattering, coherently scattered photons are detected only at specific wavelengths related to the atomic interplanar spacing $d$ of the observed material according to the Bragg law. The ideal diffraction spectrum $p(\xi)$ will thus be composed of only few Bragg peaks specific of the material crystalline structure.

Unfortunately, in practice, several effects alter the EDXRD spectrum leading to erroneous detections by fur- ther material identification algorithms. The purpose of the method presented in this paper is to recover the ideal diffraction spectrum $\boldsymbol{p}(\xi)$ from such altered measurements $\boldsymbol{y}$ by means of an inverse problems approach. To this end, we propose an linear modelization of the successive degradations leading to the data $\boldsymbol{y}$. Using matrix notation ( $\cdot$ is matrix multiplication and $\times$ element wise multiplication), this models writes:

$$
\boldsymbol{y}=\mathrm{B} \cdot[\boldsymbol{t} \times(\mathbf{H} \cdot \boldsymbol{p}+\boldsymbol{g})]+\boldsymbol{n},
$$

where:

$\mathbf{H}$ is the operator that models the blur caused by the angular distribution of the system.

$\boldsymbol{g}$ is an additional baseline spectrum caused by the amorphous part of the sample incoherent scattered radiation and multiple scattering.

$\boldsymbol{t}$ accounts for the attenuation within the sample, the non flat emission spectrum of the $\mathrm{X}$ ray tube and the fluorescence of the anode and the collimators.

$\mathbf{B}$ is the blurring operator that models the finite energy resolution of the detector.

$\boldsymbol{n}$ accounts for photon counting and detector noises.

This modelization and corresponding approximations are detailled in the next section.

\section{The direct problem: from perfect spectrum to real measurements}

\subsection{Alterations caused by the setup}

We suppose that the surface defined by the source focal spot and the detector active region are orthogonal to the $z$ axis at fixed depth of $s_{z}$ and $d_{z}$ respectively. As described on figure 1 consider a triplet $\{\boldsymbol{s}, \boldsymbol{o}, \boldsymbol{d}\}$ composed of three points $s=\left[s_{x}, s_{y}, s_{z}\right]^{\mathrm{T}}$ on the source focal spot, $\boldsymbol{o}=\left[o_{x}, o_{y}, o_{z}\right]^{\mathrm{T}}$ in the object and $\boldsymbol{d}=\left[d_{x}, d_{y}, d_{z}\right]^{\mathrm{T}}$ on the active surface of the detector. For this triplet, the scattering angle $\theta$ is defined by:

$$
\theta=\operatorname{acos}\left(\frac{\langle\overrightarrow{s \boldsymbol{s}} \cdot \overrightarrow{o d}\rangle}{\|\overrightarrow{s o}\|\|\overrightarrow{o d}\|}\right)
$$

Owing to the finite angular resolution of the setup, this angle may be different from the nominal angle $\bar{\theta}$ defined by our diffractometer. This is the cause of a blur on the spectrum. Indeed, according to the Bragg's law, a diffraction peak located at an energy $\xi$ for the nominal angle $\bar{\theta}$ will be observed at the energy $E$ for any angle $\theta$ according to the following relation:

$$
E=\xi \frac{\sin \left(\frac{\bar{\theta}}{2}\right)}{\sin \left(\frac{\theta}{2}\right)} .
$$


Conversely, for any angle $\theta$, the measured intensity at the energy $\mathrm{E}$ is proportional to the intensity of the nominal diffraction spectrum $p\left(E \frac{\sin (\theta / 2)}{\sin (\bar{\theta} / 2)}\right)$.

As consequence, after integration over the source focal spot surface $S$, the object volume $O$ and the active region of the detector $D$, the spectrum $f(E)$ measured by a perfect detector is:

$$
\begin{gathered}
f(E)=\iint_{D} \iiint_{O} \iint_{S} N(E, \boldsymbol{s}, \boldsymbol{o}) A(E, \boldsymbol{s}, \boldsymbol{o}, \boldsymbol{d}) C_{s}(\boldsymbol{s}, \boldsymbol{o}) \ldots \\
C_{d}(\boldsymbol{o}, \boldsymbol{d}) L(\theta) G(\boldsymbol{s}, \boldsymbol{o}, \boldsymbol{d}) p\left(E \frac{\sin (\theta / 2)}{\sin (\bar{\theta} / 2)}\right) \mathrm{d} \boldsymbol{s} \mathrm{d} \boldsymbol{o} \mathrm{d} \boldsymbol{d} .
\end{gathered}
$$

where:

- $N(E, \boldsymbol{s}, \boldsymbol{o})$ is the number of photons of energy $E$ emitted during the acquisition by the point $s$ of the $\mathrm{X}$ ray source in the direction of $\boldsymbol{o}$;

- $A(E, \boldsymbol{s}, \boldsymbol{o}, \boldsymbol{d})$ accounts for the attenuation at energy $E$ between $\boldsymbol{s}$ and $\boldsymbol{o}$ and between $\boldsymbol{o}$ and $\boldsymbol{d}$;

- $C_{s}(\boldsymbol{s}, \boldsymbol{o})$ and $C_{d}(\boldsymbol{o}, \boldsymbol{d})$ account for the obstruction by the source collimator and the detector collimator respectively:

$C_{i}(\boldsymbol{a}, \boldsymbol{b})= \begin{cases}0 & \text { if }(\boldsymbol{a} \boldsymbol{b}) \text { is obstructed by the collimator, } \\ 1 & \text { else, }\end{cases}$

with $i=s$ and $(\boldsymbol{a}, \boldsymbol{b})=(\boldsymbol{s}, \boldsymbol{o})$ or $i=d$ and $(\boldsymbol{a}, \boldsymbol{b})=$ $(\boldsymbol{o}, \boldsymbol{d})$

- $L(\theta)$ is the Lorentz polarization factor[11];

- $G(\boldsymbol{s}, \boldsymbol{o}, \boldsymbol{d})$ is a geometrical factor depending only on distances between the setup elements:

$$
G(s, o, d)=\frac{1}{\|\overrightarrow{s o}\|^{2}\|\overrightarrow{o d}\|}
$$

In some cases, if the object is not too extended, it is possible to assume that Lorentz polarization and geometrical factors are constant. In order to cope with such equation, several simplifications are described in the next subsection.

\subsection{Attenuation and source spectrum}

The source is supposed to emit uniformly over the focal spot and this emission is supposed to be isotropic. Therefore, we can define $N(E)$ the number of photons emitted during the acquisition. When the source is an $\mathrm{X}$ ray tube, the source spectrum $N(E)$ is a bremsstrahlung spectrum with fluorescence lines depending of the $\mathrm{X}$ ray tube anode.

The attenuation between $\boldsymbol{s}, \boldsymbol{o}$ and $\boldsymbol{d}$ follows the BeerLambert law:

$A(E, \boldsymbol{s}, \boldsymbol{o}, \boldsymbol{d})=\exp \left(-\int_{\boldsymbol{s}}^{\boldsymbol{o}} \mu(E, \ell) \mathrm{d} \ell-\int_{\boldsymbol{o}}^{\boldsymbol{d}} \mu(E, \ell) \mathrm{d} \ell\right)$,

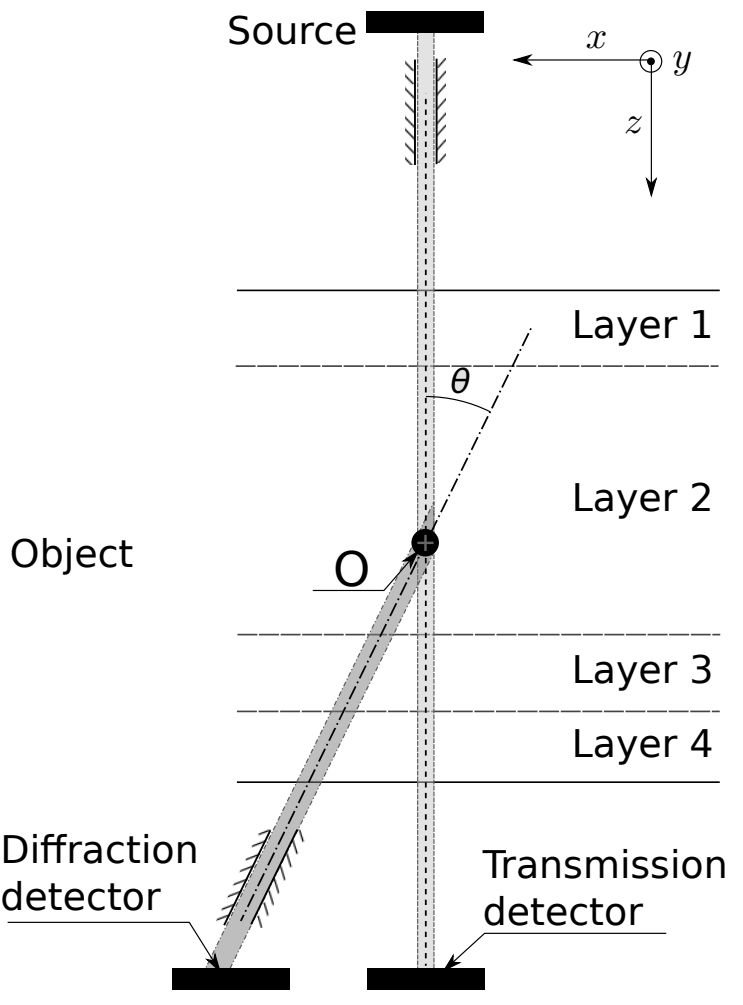

Figure 2: Schematic view of a $\mathrm{X}$ ray diffraction setup and notations used in the model.

where $\mu(E, \ell)$ is the linear attenuation of the material at position $\ell$ at energy $E$. If the probed volume dimension along the $x$ and $y$ axis are smaller than the morphological structures in the observed object, it can be considered as a succession of layers of different linear attenuation $\mu_{i}(E)$ and thickness $L_{i}$ orthogonal to the $z$ axis as depicted on figure 2. Moreover, as the scattering angle $\theta$ and the angular aperture of collimators $\alpha$ are sufficiently small, it is possible to hold the classical small angle approximations $\cos (\theta) \approx 1$ and as proposed by Harding[12-14]. The attenuation term thus simplifies to the transmission attenuation:

$$
A(E)=\exp \left(-\sum_{i} \mu_{i}(E) L_{i}\right)
$$

With such approximations, the product $t(E)=$ $N(E) A(E)$ represents the number of transmitted photons which may be estimated using a spectroscopic detector placed in transmission direction[12].

\subsection{Model of the geometrical blur}

As the transmitted spectrum $t(E)$ depends only on the energy, it can be factorized and removed from the integration in equation 5. As consequence, in this integral only the term $p\left(E \frac{\sin (\theta / 2)}{\sin (\bar{\theta} / 2)}\right)$ depends on energy. 


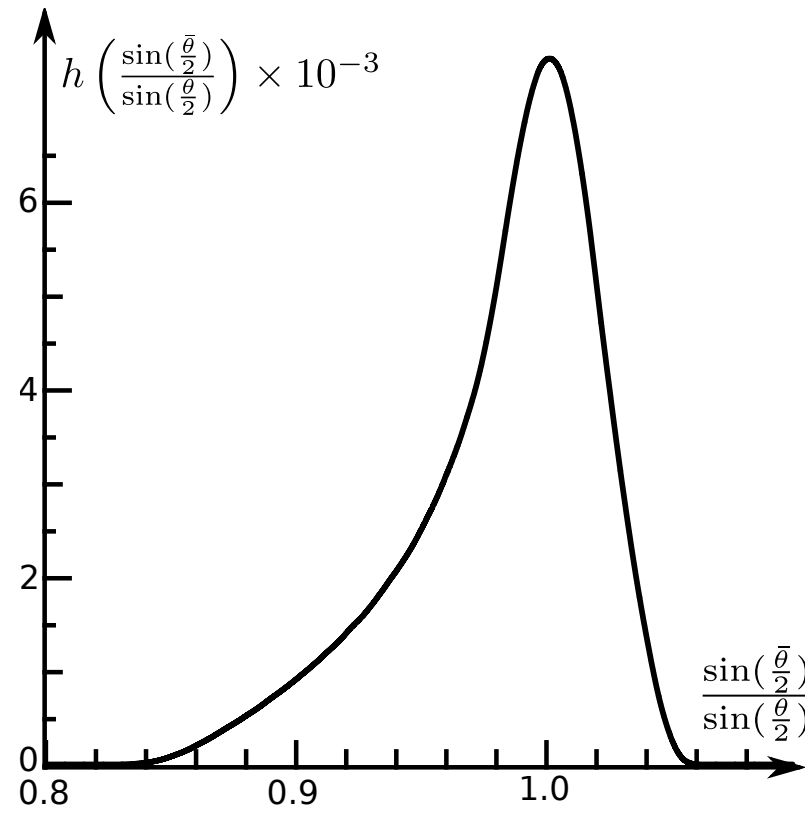

Figure 3: Density $h\left(\frac{\sin (\bar{\theta} / 2)}{\sin (\theta / 2)}\right)$ of the angular distribution of the experimental setup presented at sections 6.1 and 6.3

We define the kernel function $h()$ :

$h\left(\frac{\sin (\bar{\theta} / 2)}{\sin (\theta / 2)}\right)=\int_{\Gamma(\theta)} C_{s}(\boldsymbol{s}, \boldsymbol{o}) C_{d}(\boldsymbol{o}, \boldsymbol{d}) L(\theta) G(\boldsymbol{s}, \boldsymbol{o}, \boldsymbol{d}) \mathrm{d} \ell$.

where $\Gamma(\theta)$ is the contour defined by $\widehat{\boldsymbol{s o d}}=\theta$ and $\mathrm{d} \ell$ an elementary part of this contour. This function is a weighted density where $h\left(\frac{\sin (\bar{\theta} / 2)}{\sin (\theta / 2)}\right)$ is length of the iso-contours defined by $\widehat{\boldsymbol{s o d}}=\theta$ in the seven dimensions volume $S O D$. These contours are weighted by the geometric, Lorentz polarization and collimators functions.

According to the Bragg law, two couples $\{E, \theta\}$ and $\left\{E^{\prime}, \theta^{\prime}\right\}$ probing the same inter-planar spacing $d$ are linked by the equation 4 . We thus have:

$$
h\left(\frac{\sin (\bar{\theta} / 2)}{\sin (\theta / 2)}\right)=h\left(\frac{E}{\xi}\right) .
$$

The geometric blurring effect caused by the configuration of the setup can be therefore expressed by a simple integral as we can rewrite equation 5 :

$$
f(E)=t(E) \int h\left(\frac{E}{\xi}\right) p(\xi) \mathrm{d} \xi .
$$

We recognize a Fredholm integral equation of the first kind.

Kernel numerical estimation. This equation cannot be analytically solved and we proposed to use a Monte Carlo integration to provide a good numerical estimation of it. Monte Carlo should be less prone to artifacts than Riemann sum proposed by Langford[10, 14]. This method consists on picking randomly a large number of septuplet $\left\{s_{x}, s_{y}, o_{x}, o_{y}, o_{z}, d_{x}, d_{y}\right\}$. For each septuplet, if both collimator functions are equal to 1 , we compute the angle $\theta$ and the geometric and Lorentz polarization functions. The density of $\frac{\sin (\bar{\theta} / 2)}{\sin (\theta / 2)}$ weighted by geometrical and Lorentz polarization functions is then estimated using a ParzenRosenblatt window method with an Epanechnikov window REFS. An exemple of such histogram computed for experimental conditions given at sections 6.1 and 6.3 is shown on figure 3 . This density presents an important negative skewness and cannot be easily approximated by distribution functions classically used in ADXRD peak fitting algorithms (such as Gaussian, Cauchy, Pearson... ) [3].

\subsection{Background}

During the acquisition, the detector measures not only blurred diffraction peaks but also an additional baseline spectrum. This spurious background $g(E)$ level is caused by amorphous parts of the sample, incoherent scattered radiation and multiple scattering.

$$
m(E)=t(E)(f(E)+g(E)) .
$$

\subsection{Acquisition}

The detector measures the number of photons in a discrete number of energy channels. These measurements $\boldsymbol{y}$ are inevitably corrupted by some noise $\boldsymbol{n}$. There are mainly three sources of noise:

- photon counting noise which follows a Poisson law,

- thermal noise which is a Gaussian noise,

- modelization noise which is use to take into account approximations made to define the physical direct model. We suppose this noise Gaussian.

For a channel $j$ centered in $E_{j}$, the detector has a spectral response $b^{(j)}(E)$ and the measurement $y_{j}$ in this channel is:

$$
y_{j}=\int t(E)(f(E)+g(E)) b^{(j)}(E) \mathrm{d} E+n_{j},
$$

If the response is the same for all channels $\left(b^{(j)}(E)=\right.$ $\left.b\left(E-E_{j}\right)\right)$, the equation 14 is a convolution. In practice the support of $b^{(j)}(E)$ is larger than the energy step between two consecutives channels, as consequence it causes spectral blurring.

\subsection{Discretization}

Without loss of generality, the spectral distributions $t(E), g(E)$ and $p(\xi)$ can be parametrized by a finite number of parameters by means of expansion onto a basis of interpolation functions (REF). As the measurements $\boldsymbol{y}$ are by definition discrete, it is natural to use the same evenly spaced spectral grid $\mathcal{G}_{E}=\left\{\stackrel{\mathrm{a}}{E_{\ell}} ; \ell=1, \ldots, N_{E}\right\}$ to discretize $t(E), g(E)$ and the spectral response functions $b^{(j)}(E)$. In this case, there is no need to explicitly choose 
any particular basis of interpolation functions as far as it has the properties required to make them suitable as interpolation functions and it comes:

$$
\begin{aligned}
t_{i} & =t\left(\stackrel{\mathrm{a}}{E}_{i}\right) \\
g_{i} & =g\left(\stackrel{\mathrm{a}}{E}_{i}\right) \\
b_{i}^{(j)} & =b^{(j)}\left(\stackrel{\mathrm{a}}{E}_{i}\right)
\end{aligned}
$$

For the parameter $p(\xi)$ which is the one we want to extract, more attention has to be paid on discretization. It can be discretized on another spectral grid $\mathcal{G}_{\epsilon}=\left\{\stackrel{\mathrm{D}}{\epsilon}_{j} ; j=\right.$ $\left.1, \ldots, N_{\epsilon}\right\}$ using the interpolation function $v_{j}(\epsilon)$

$$
p(\xi)=\sum_{j} p_{j} v_{j}\left(\xi-\stackrel{a}{\epsilon}_{j}\right) .
$$

The grid $\mathcal{G}_{\epsilon}$ can be the same as $\mathcal{G}_{E}$ but can also be more finely discretized to perform super-resolution. Furthermore, it may not be necessary evenly spaced and can be designed to be evenly spaced in the momentum transfer space $\left(\dot{x}_{j}=\stackrel{ᄆ}{\epsilon}_{j} \frac{\sin (\bar{\theta} / 2)}{h c}\right)$ or in the angle space for a given energy $\bar{E}$ in order to be directly compared with a existing database indexed in momentum transfer or in angle. In this work, we choose to use the grid of the measurements: $\mathcal{G}_{\epsilon}=\mathcal{G}_{E}\left(\stackrel{\square}{\epsilon}_{j}=\stackrel{\mathrm{E}}{E}_{j}\right)$ and to define $v_{j}(\epsilon)=v(\epsilon)$ as uniform:

$$
v(\epsilon)= \begin{cases}\frac{1 .}{\kappa} & \text { if }-\frac{\kappa}{2} \leq \epsilon<\frac{\kappa}{2} \\ 0 & \text { otherwise }\end{cases}
$$

where $\kappa$ is the channel width of the detector.

Using such parametrization our discrete model writes:

$$
y_{i}=\sum_{j} B_{i, j} t_{j}\left(g_{j}+\sum_{k} H_{j, k} p_{k}\right)+n_{i} .
$$

where the coefficients of $B_{i, j}$ and $H_{j, k}$ are:

$$
\begin{aligned}
B_{i, j} & =b^{(i)}\left(\stackrel{\mathrm{a}}{E}_{j}\right) \\
H_{j, k} & =\int_{\mathbb{R}^{+*}} h\left(\frac{\stackrel{\mathrm{a}}{E}_{j}}{E^{\prime}}\right) v\left(E^{\prime}-\stackrel{\mathrm{a}}{\epsilon}_{k}\right) \mathrm{d} E^{\prime} .
\end{aligned}
$$

Equation 20 can be written using matrix notation and becomes the direct discrete:

$$
\boldsymbol{y}=\mathbf{B} \cdot[\boldsymbol{t} \times(\mathbf{H} \cdot \boldsymbol{p}+\boldsymbol{g})]+\boldsymbol{n} .
$$

This equation defines our direct problem and thus can be used in order to simulate the data. In practice filter reponse $b^{(i)}$ remains the same for all channels and the convolution matrix $\mathbf{B}$ can be fastly applied by means of a Fourier transform. A simulation using this model with $\boldsymbol{g}=0$ and $\boldsymbol{p}=0$ excepted at $30 \mathrm{keV}, 80 \mathrm{keV}$ and $140 \mathrm{keV}$ is shown on figure 4 for experimental conditions given at sections 6.1 and 6.3. On this figure, it can be clearly seen that the spread of the peaks increases as their energy rises.

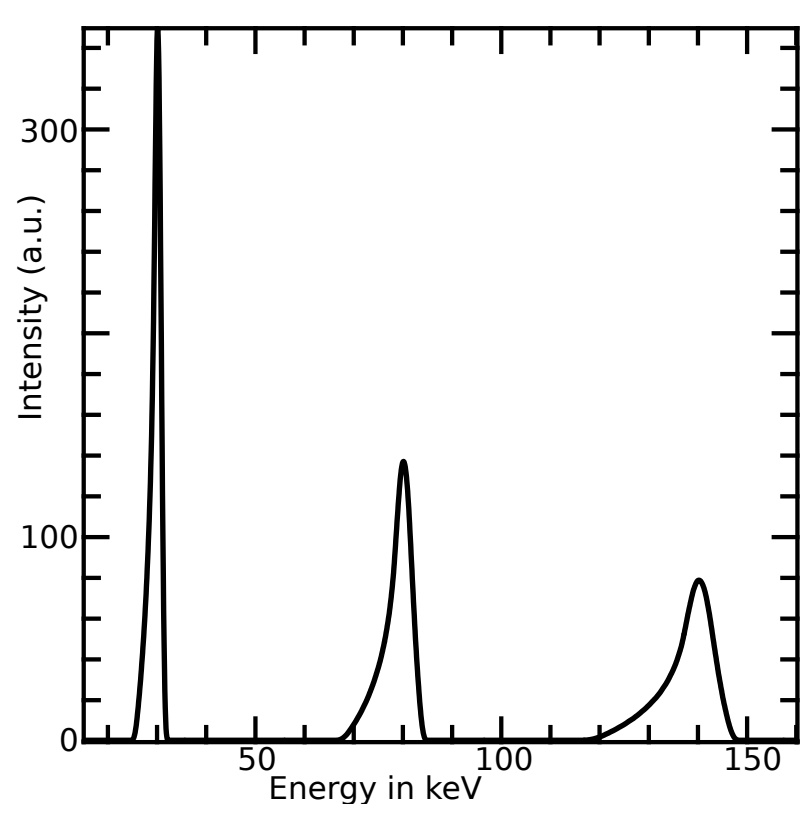

Figure 4: Total response of the setup for three peaks of equal intensity placed at $30 \mathrm{keV}, 80 \mathrm{keV}$ and $140 \mathrm{keV}$.

\section{The inverse problem:}

The goal of the presented work is to estimate the diffraction peak distribution $\boldsymbol{p}$ from the data $\boldsymbol{y}$ according to the model defined by equation 2 . We suppose that the operators $\mathbf{B}$ and $\mathbf{H}$ are known and that a good estimation of $\boldsymbol{t}$ is provided by measurements from a spectroscopic detector placed in transmission. However, even if it is not of interest, we still have to estimate the background $\boldsymbol{g}$ jointly with $\boldsymbol{p}$ (both of size $N$ ) from a data spectrum of size $N$. This is a typical inverse problem, that can be solved in a penalized likelihood or maximum a posteriori (MAP) framework [15].

This is achieved by estimating the couple $\left\{\boldsymbol{p}^{+}, \boldsymbol{g}^{+}\right\}$that minimizes the cost function $\xi(\boldsymbol{f}, \boldsymbol{g})$ :

$$
\begin{aligned}
\left\{\boldsymbol{p}^{+}, \boldsymbol{g}^{+}\right\} & =\underset{\{\boldsymbol{p}, \boldsymbol{g}\}}{\arg \min } \xi(\boldsymbol{p}, \boldsymbol{g}), \\
\xi(\boldsymbol{p}, \boldsymbol{g}) & =\Phi(\boldsymbol{p}, \boldsymbol{g}, \boldsymbol{y})+\Psi(\boldsymbol{p})+\Theta(\boldsymbol{g}),
\end{aligned}
$$

This cost function $\xi(\boldsymbol{p}, \boldsymbol{g})$ is the sum of three terms: a likelihood penalty $\Phi(\boldsymbol{p}, \boldsymbol{g} ; \boldsymbol{y})$ ensuring the agreement between the model defined by equation 2 and the data $\boldsymbol{y}$, and two regularization penalties $\Psi(\boldsymbol{p})$ and $\Theta(\boldsymbol{g})$ introducing subjective a priori knowledge about the diffraction peak distribution $\boldsymbol{p}$ and the background $\boldsymbol{g}$ respectively.

\subsection{The likelihood penalty term}

Assuming Gaussian noise, the likelihood penalty reads:

$$
\Phi(\boldsymbol{p}, \boldsymbol{g})=\boldsymbol{r}^{\mathrm{T}} \cdot \mathbf{W} \cdot \boldsymbol{r}
$$

with the residuals:

$$
\boldsymbol{r}=\boldsymbol{y}-\mathbf{B} \cdot[\boldsymbol{t} \times(\mathbf{H} \cdot \boldsymbol{p}+\boldsymbol{g})]
$$


and where the weighting matrix $\mathbf{W}$ is the inverse of the covariance of the noise: $\mathbf{W}=\mathbf{C}_{\text {noise }}^{-1}$.

If the noise is uncorrelated, the weighting matrix is diagonal $\mathbf{W}=\operatorname{diag}(\boldsymbol{w})$ and Eq. 26 simplifies to:

$$
\Phi(\boldsymbol{p}, \boldsymbol{g})=\sum_{i} w_{i} r_{i}^{2}
$$

where $1 / w_{i}$ is the noise variance in the channel $i$. This model can cope with non-stationary noise and can be used to express confidence on measurements on each spectral channel of the data. Since unmeasured data have infinite variance, we can readily deal with missing (outside the field of view) or bad channels as follows:

$$
w_{i} \equiv \begin{cases}\operatorname{Var}\left(y_{i}\right)^{-1} & \text { if } y_{i} \text { is measured } \\ 0 & \text { otherwise }\end{cases}
$$

Except for very low detector noise, we can approximate the total noise (Gaussian detector noise plus Poissonnian signal noise) by a non stationary Gaussian noise [16]:

$$
w_{i} \equiv \begin{cases}\left(\gamma \max \left(y_{i}, 0\right)+\sigma_{i}^{2}\right)^{-1} & \text { if } y_{i} \text { is measured } \\ 0 & \text { otherwise }\end{cases}
$$

where $\gamma$ accounts for the quantization factor of the detector and $\sigma_{i}^{2}$ is the variance of other approximately Gaussian noise (for example read-out noise) in the channel $i$.

\subsection{Priors}

The most evident objective prior on interference functions we can introduce is its positivity. Unfortunately this constraint is not strong enough to lead to acceptable solutions, and other a priori knowledge are needed. These priors are introduced by the use of the regularization functions $\Psi(\boldsymbol{p})$ and $\Theta(\boldsymbol{g})$. As the diffraction peak distribution $\boldsymbol{p}$ and the background $\boldsymbol{g}$ have very different a priori morphological shape, these two functions are designed separately.

Prior on the diffraction peaks distribution. Interference function of a crystalline material is non-null only for values that correspond to the inverse of crystal inter-planar spacing. The diffraction peak distribution $\boldsymbol{p}$ is then very sparse. Recovery of sparse vectors has been extensively studied in this last decade ${ }^{1}$. Donoho [17] has shown that minimizing the $\ell_{1}$ norm of a vector $\boldsymbol{p}$ is the best convex regularization function that favor sparsest solutions. Unfortunately, $\ell_{1}$ norm is not continuously derivable preventing fast convergence of our continuous optimization procedure. For that reason we use a continuously derivable hyperbolic approximation defined by:

$$
\Psi(\boldsymbol{p})=\sum_{k} \alpha_{k}\left[\sqrt{p_{k}^{2}+\varepsilon^{2}}-\varepsilon\right]
$$

where $\varepsilon$ is very small $\left(\approx 10^{-9}\right)$ and $\alpha_{k}$ are the so-called hyper-parameters that may vary with the channel $k$.

\footnotetext{
${ }^{1}$ The website http://www.compressedsensing.com/ contains many references about sparsity and compressive sensing.
}

Priors on the background. As we don't have precise (i.e. quantitative) information about the main effects that lead its formation, we propose to introduce some smoothness priors about the background $\boldsymbol{g}$. The background regularization function $\Theta(\boldsymbol{g})$ is thus a classical quadratic smoothness regularization function defined by:

$$
\Theta(\boldsymbol{g})=\sum_{k} \beta_{k}\left(g_{k+1}-g_{k}\right)^{2}
$$

where $\beta_{k}$ are channel varying hyper-parameters. This regularization function will act as a low pass filter which prevent sharp changes of the interference function.

\subsection{Hyper-parameters settings}

$\alpha_{k}$ and $\beta_{k}$ are the hyper-parameters that are used to balance the influence of both priors and likelihood and that have to be properly tuned. Unfortunately, as these hyper-parameters are channel varying, we replace an estimation problem with $2 N$ parameters by another problem of $(2 N-1)$ hyper-parameters estimation. However, the large number of hyper-parameters defined in this very general scheme can be drasticly reduced according to simple rules.

In this work, the parameter of interest is the diffraction peak distribution $\boldsymbol{p}$. Without informations about the material nature, the probability of presence of a diffraction peak is uniformly distributed over the channels. Unfortunately, constant hyper-parameters $\alpha_{k}$ and $\beta_{k}$ favor diffraction peaks at low energy. Indeed, as shown in figure 4 , in the data $\boldsymbol{y}$, diffraction peaks at low energy are sharper than those at high energy and a sharp peak has a higher probability to be correlated with some noise realization than a smooth one. The hyper-parameters $\alpha_{k}$ have thus to be tuned to enforce an uniform distribution of the diffraction peaks. Mimicking the L2 normalisation of atoms of a dictionnary used in matching pursuit algorithms[18], we propose a normalization of the hyper-parameter $\alpha_{k}$ by the L2 norm of the corresponding blurred peaks. It writes thus:

$$
\alpha_{k}=\alpha \sqrt{\sum_{i}\left[\sum_{j} B_{i, j} H_{j, k}\right]^{2}},
$$

where $\alpha$ is a global hyper-parameter for diffraction peak distribution regularization.

Furthermore, the background regularization $\Theta(\boldsymbol{g})$ favors smooth background. As a peak at high energy is smooth, the hyper-parameters $\beta_{k}$ must be tuned to enforce a background smoother than diffraction peaks everywhere in the spectrum and to enforce an uniform distribution of the distribution peaks.

$$
\beta_{k}=\beta \frac{1}{\sum_{i}\left[\nabla\left(H_{-, i}\right)\right]_{k}^{2}}
$$

where $H_{-, i}$ means the i-th column of $\mathbf{H}$ and $\nabla$ is the finite difference operator. $\beta$ is a global hyper-parameter for the 
background regularization. This normalization of $\beta_{k}$ ensure that the regularization function $\Theta(\boldsymbol{g})$ gives the same penalty for all peaks wherever their position is in the spectrum. Finaly only two hyper-parameters $\beta$ and $\alpha$ have to be tuned for the reconstruction.

\section{Algorithm summary}

In our framework, the reconstruction of the diffraction peak distribution $\boldsymbol{p}$ and the background $\boldsymbol{g}$ corresponds to the determination of the couple $\left\{\boldsymbol{p}^{+}, \boldsymbol{g}^{+}\right\}$that minimizes the objective criterion defined in Eq. 25. The optimal reconstructed diffraction peak distribution depends on the particular values of the hyper-parameters $\alpha$ and $\beta$. Choosing their optimal values is difficult and it is done for now by trials and errors. However, for future automatic hyperparameters estimation, the L-curve method[19] or Stein unbiased risk estimator [20] seems suitable, but this deserves an extensive study which is out of the scope of this paper.

To minimize the criterion defined in Eq. 25., we use the VMLM-B (Variable Metric with Limited MemoryBounded) algorithm [21] which is a limited memory variant of the variable metric method with BFGS updates [22]. This algorithm can further account for bound constraints on the parameters. We make use of this feature to enforce positivity of $\boldsymbol{p}$ and $\boldsymbol{g}$. This algorithm has proved its effectiveness for image reconstruction and only requires the computation of the penalty function being minimized and its gradient. The memory requirement is a few times the size of the problem.

\section{Results}

In this section, we illustrate the performance of the proposed algorithm using real data. Two crystalline materials with well known inter-planar spacing (since [23, 24]) are used in these experiments: $\mathrm{NaCl}$ and graphite.

\subsection{Experimental setup}

Schematic representation of the experimental setup is shown in figure 5 . The sample is illuminated by a $140 \mathrm{kV}$ tungsten anode X-ray tube operated at $280 \mu \mathrm{A}$ and placed at $36.7 \mathrm{~cm}$ from the sample. Its focal spot is $1 \mathrm{~mm}$ wide. The incident X-ray beam is collimated by a tungsten slit of $0.2 \mathrm{~mm}$ in width, $8 \mathrm{~mm}$ in height and $8 \mathrm{~cm}$ in length. This source collimator is placed $18.4 \mathrm{~cm}$ away from the focal spot.

The scattered photons are detected with an high purity germanium (HPGe) detector oriented at $\bar{\theta}=4.26^{\circ}$ and placed $24 \mathrm{~cm}$ from the sample. The detector collimator located in front of the detector is a slit of $8 \mathrm{~mm}$ in height and $8 \mathrm{~cm}$ in length. This slit has a width of $0.02 \mathrm{~mm}$ on the detector side and $0.2 \mathrm{~mm}$ on the other side. The spectral response function of the HPGe detector energy $\boldsymbol{b}$ is supposed to be a Gaussian with a full width at half

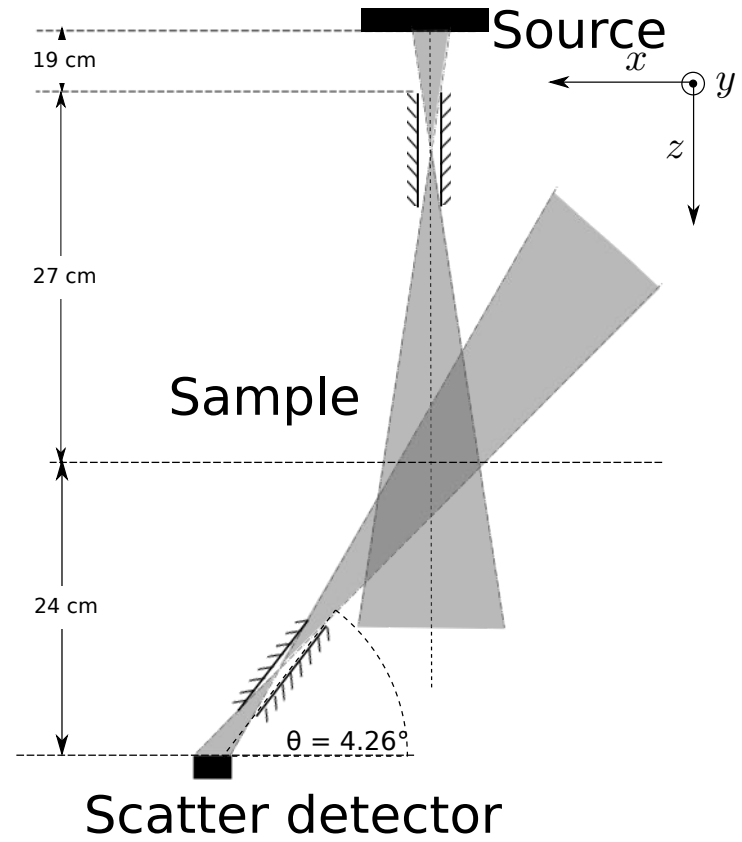

Figure 5: Schematic view of the setup used in the experiments.

maximum (FWHM) of $0.4 \mathrm{keV}$. It is supposed to be constant on the whole energy range considered in these experiments: $\boldsymbol{b}^{(j)}=\boldsymbol{b}$. We consider every photon detected in $N_{L}=1818$ energy channels of the detector between $15 \mathrm{keV}$ and $160 \mathrm{keV}$ (sampling step size of $80 \mathrm{eV}$ ). With such a couple source/detector the range of the probed interplanar spacing is beetwen $0.2 \mathrm{~nm}^{-1}$ and $2.4 \mathrm{~nm}^{-1}$. The exposure time is set at $600 \mathrm{~s}$, for each spectra to have a good counting statistic.

In both experiments, the matrix $\mathbf{H}$ is computed according to the equation 22 using these setup parameters and the approximative shape of the sample. Without transmission detector, the source spectrum was approximated by a Birch and Marshall[25] semi empirical spectrum for a tungsten anode $160 \mathrm{kV}$ source. For the attenuation spectrum we use the theoretical attenuation of the samples.

\section{2. $\mathrm{NaCl}$}

The $\mathrm{NaCl}$ used was held in a thin walled polyethylene cylinder of $1 \mathrm{~cm}$ diameter and $2 \mathrm{~cm}$ in height. The scattering measurements are presented on figure 6 . Several comments can be made on these data and we can notice:

- a continuous background that is higher for low energy,

- four peaks that may be produced by diffraction at $50 \mathrm{keV}, 59 \mathrm{keV}, 83 \mathrm{keV}$ and $102 \mathrm{keV}$,

- two peaks at $59.7 \mathrm{keV}$ and $67 \mathrm{keV}$ that are caused by tungsten fluorescence,

- a very broad peak between $30 \mathrm{keV}$ and $40 \mathrm{keV}$ which may be due to the scattering of the plastic cylinder. 


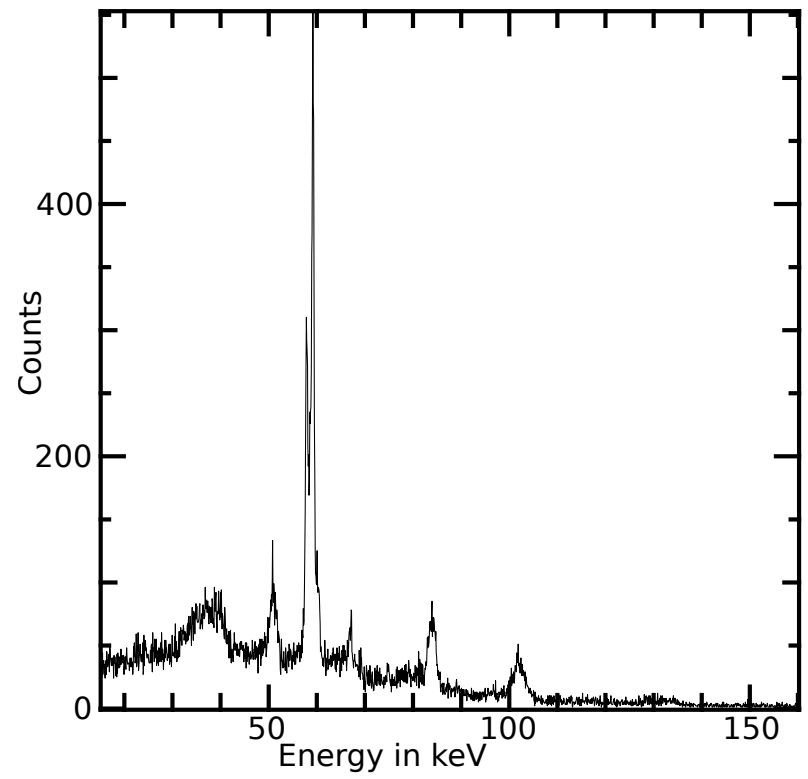

Figure 6: Measurements on a $\mathrm{NaCl}$ sample.

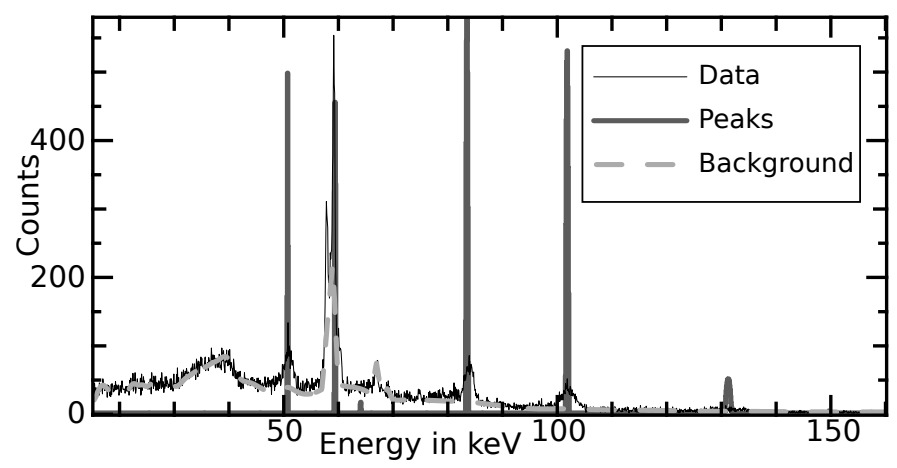

Figure 7: $\mathrm{NaCl}$ diffraction spectrum (in black) with the coresponding estimated background $\boldsymbol{g} \times \boldsymbol{t}$ (in dashed light gray) and diffraction peaks $\boldsymbol{p}$ (in dark gray).

This peak is too broad to be caused by scattering of crystalline materials.

These data were processed using the proposed algorithm with hyper parameters $\alpha=0.05$ and $\beta=10$. The source spectrum was supposed to follow the Birch and Marshall synthetic spectrum and we use the theoretical attenuation of a $1 \mathrm{~cm}$ thick $\mathrm{NaCl}$ cube. Estimated diffraction peak distribution $\boldsymbol{p}$ and background $\boldsymbol{g}$ are shown on figure 7 .

As expected the estimated background $\boldsymbol{g}$ fits the broad peak between $30 \mathrm{keV}$ and $40 \mathrm{keV}$ but not the next peak as $50 \mathrm{keV}$ which is a diffraction peak. As the background plotted on figure 7 is the product $\boldsymbol{g} \times \boldsymbol{t}$, the peaks about $58 \mathrm{keV}$ and $67 \mathrm{keV}$ correspond to the Tungsten fluorescence lines.

The diffraction peak distribution $\boldsymbol{p}$ shows six peaks. The positions of the centroid of these peaks are presented in the table 1 . They can be compared with the theoretical diffraction peak positions estimated for $\bar{\theta}=4.26^{\circ}$ using inter-planar spacing measured by Swanson[26] (from

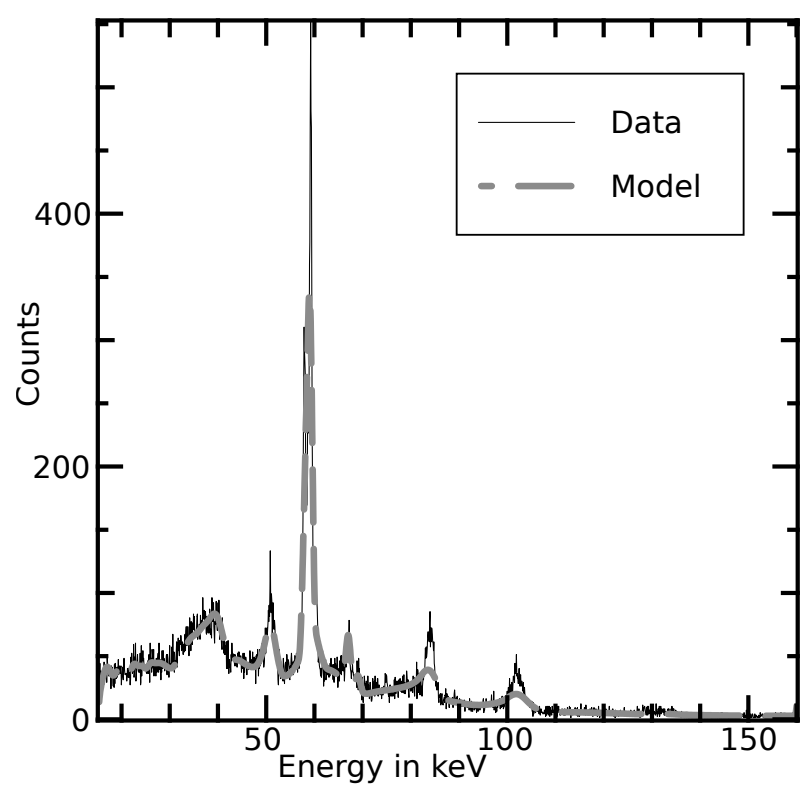

Figure 8: $\mathrm{NaCl}$ diffraction spectrum (in black) and its model (in light gray) computed using estimated vectors $\boldsymbol{p}$ and $\boldsymbol{g}$

\begin{tabular}{|cccc|c|}
\hline \multicolumn{4}{|c|}{ Theory (from [26]) } & Estimation \\
\hline hkl & d in $\AA$ & $\begin{array}{c}I / I_{\max } \\
\text { in } \%\end{array}$ & $\begin{array}{c}\text { E at } \theta=4.26^{\circ} \\
\text { in } \mathrm{keV}\end{array}$ & $\begin{array}{c}\text { peak position } \\
\text { in keV }\end{array}$ \\
\hline 111 & 3.258 & 13 & 51.20 & 50.75 \\
200 & 2.821 & 100 & 59.13 & 59.42 \\
& & & false & 64.10 \\
220 & 1.994 & 55 & 83.65 & 83.54 \\
311 & 1.701 & 2 & 98.06 & ND \\
222 & 1.628 & 15 & 102.45 & 101.85 \\
400 & 1.410 & 6 & 118.29 & ND \\
331 & 1.294 & 1 & 128.90 & ND \\
420 & 1.261 & 11 & 132.27 & 131.29 \\
422 & 1.1515 & 7 & 144.85 & ND \\
511 & 1.0855 & 1 & 153.66 & ND \\
\hline
\end{tabular}

Table 1: Estimated diffraction peak position (shown on Fig. 7) compared to position for $\bar{\theta}=4.26^{\circ}$ estimated using inter-plannar spacing measurements from from [26]. false is for false detection and $N D$ for non detection. The theoretical position of the peaks have been estimated for $\bar{\theta}=4.26^{\circ}$ 
ASTM card 5-0628). Every peaks present a very accurate position compared to the Swanson estimation with an error smaller than $1 \mathrm{keV}$. The last detected peak (at $131.29 \mathrm{keV}$ ) presents the higher positioning error which can be explained by the relatively small number of photons measured around this energy. The five diffraction peaks that were not detected, are the five peaks with the lower relative intensity $(<10 \%)$ in the measurements of Swanson. A small peak has been falsely detected at $64.1 \mathrm{keV}$. This false detection may be due to the tungsten fluorescence line $K_{\beta}$ at $67.25 \mathrm{keV}$. Indeed, as we use only a very coarse estimation of both source spectrum and material attenuation, the magnitude of these rays may be under-estimated, and their residuals may be explained as a scattering line by our algorithm. This problem of too coarse estimation of source spectrum and material attenuation is responsible for the misestimation of peak magnitude. Furthermore as the presented method is for powder diffraction, multiplicity and texture (prefered orientation of crystal) may affect measured relative intensity. As consequence, only peak's positions can be used for material identification and not relative intensity.

The figure 8 shows the model of the data computed with estimated solutions $\boldsymbol{p}$ and $\boldsymbol{g}$. This model shows a good accordance with the data estiblishing the validity of our linear model of the formation of EDXRD data. However, it can be noticed that the model is always lower than data around the diffraction peaks. This under estimation can be explained by a too coarse estimation of the setup geometry that has lead to an over estimation of the angular dispersion giving thus too broad diffraction peaks.

\subsection{Graphite}

The graphite used in this experiment was a $4 \mathrm{~cm}$ thick piece of about $2 \mathrm{~cm}$ in width and height. This large size of the sample is responsible for quite broad scattering peaks in the scattering measurements. This EDXRD diffraction spectrum is presented on figure 9. Data were processed using the proposed algorithm with hyper parameters $\alpha=4.10^{-3}$ and $\beta=5$. We use the theoretical attenuation of a $4 \mathrm{~cm}$ thick graphite piece. The results of the algorithm is plotted on figure 10 and the model of the data computed with estimated solutions $\boldsymbol{p}$ and $\boldsymbol{g}$ is plotted on figure 11. This figure shows the accordance between the model and the data. The estimated locations of diffraction peaks are shown in the table 2 and can be compared with "theoretical" peaks position estimated using inter-planar spacing measurements given by ASTM card 12-212 [27].

On figure 10, we can see that seven diffraction peaks are detected. Most of them (excepted the highest peak at $49.11 \mathrm{keV}$ ) were difficult to distinguish on the data (figure 9), in particular for those at energy higher than $120 \mathrm{keV}$ where very few photons $(<5)$ are detected in a single channel. Only the three fainter peaks were not detected. Furthermore, the $K_{\alpha}$ line of the tungsten (at $58.9 \mathrm{keV})$ is not identified as a diffraction peak. The highest peak at $49.11 \mathrm{keV}(h k \ell: 002)$ has two visible harmonics

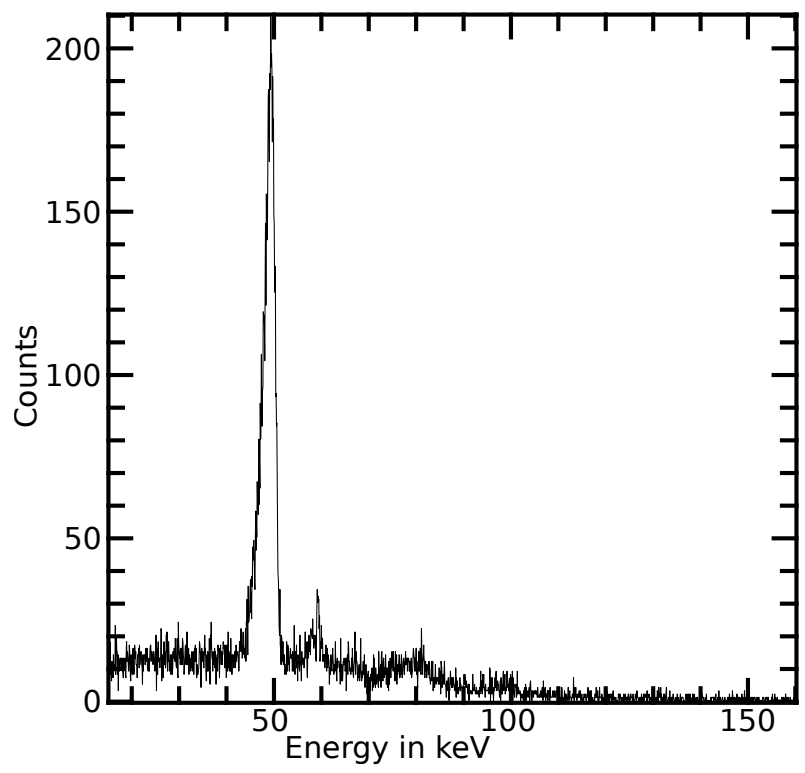

Figure 9: EDXRD measurements on a Graphite sample.

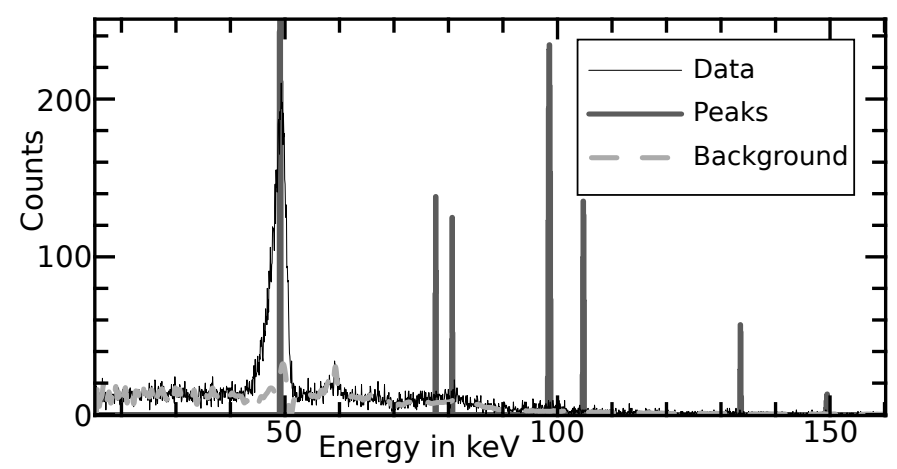

Figure 10: Graphite diffraction spectrum (in black) with the coresponding estimated background $\boldsymbol{g} \times \boldsymbol{t}$ (in dashed light gray) and diffraction peaks $\boldsymbol{p}$ (in dark gray).

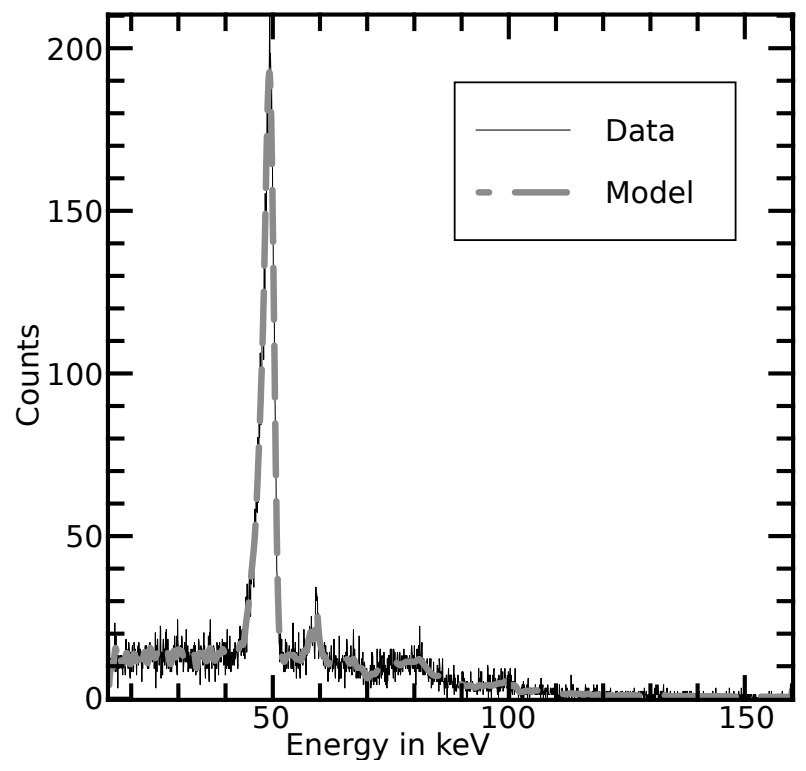

Figure 11: Graphite diffraction spectrum (in black) and its model (in dashed light gray) computed using estimated vectors $\boldsymbol{p}$ and $\boldsymbol{g}$ 


\begin{tabular}{|c|c|c|c|c|c|}
\hline \multicolumn{4}{|c|}{ Theory (from [27]) } & \\
\hline hkl & $\mathrm{d}$ in $\AA$ & $\begin{array}{l}I / I_{\max } \\
\text { in } \%\end{array}$ & $\begin{array}{c}\mathrm{E} \text { at } \bar{\theta}=4.26^{\circ} \\
\text { in } \mathrm{keV}\end{array}$ & $\begin{array}{l}\text { peak positi } \\
\text { in } \mathrm{keV}\end{array}$ & $\begin{array}{l}\text { iomly peaks location could be used. This coarse estimation } \\
\text { is also responsible of false detections around fluorescence }\end{array}$ \\
\hline 002 & 3.370 & 100 & 49.49 & 49.11 & line of the tungsten. These drawback should be attenu- \\
\hline 100 & 2.132 & 2 & 78.23 & 77.66 & ated by the simultaneous acquisition of the transmission \\
\hline 101 & 2.036 & 3 & 81.92 & 80.69 & spectrum that should give (in the small angle approxima- \\
\hline 102 & 1.800 & 1 & 92.66 & ND & tion) a better estimation of the attenuation times source \\
\hline $004^{*}$ & 1.682 & 8 & 99.16 & 98.50 & spectrum. \\
\hline 103 & 1.541 & 2 & 108.24 & 104.76 & The other sticking point of the presented method is the \\
\hline 110 & 1.232 & 6 & 135.34 & 133.58 & necessity to have at least a coarse estimation of the shape \\
\hline 112 & 1.155 & 2 & 144.41 & ND & of the sample to compute the blurring kernel $h()$. A way to \\
\hline $006^{*}-105$ & 1.120 & 2 & 148.92 & 149.47 & bypass this shape estimation step is to directly calibrate \\
\hline 201 & 1.050 & 2 & 158.85 & ND & this kernel $h()$ using the measurements as it is done in \\
\hline $\begin{array}{l}\text { the peak at } 4 \\
\text { detection. }\end{array}$ & & a & 等 & $\begin{array}{l}\text { tion } \\
\text { sof } \\
\text { non }\end{array}$ & $\begin{array}{l}\text { image blind deconvolution algorithm[29]. } \\
\text { A natural extension of this "inverse problem" is the re- } \\
\text { construction of a } 3 \mathrm{D} \text { volume using an array of detectors } \\
\text { and the estimation of a whole } 3 \mathrm{D} \text { crystalline material as }\end{array}$ \\
\hline
\end{tabular}

at $98.50 \mathrm{keV}$ ( $h k \ell: 004)$ and $149.47 \mathrm{keV}$ ( $h k \ell: 006)$. The baseline of the diffraction spectrum is well fitted by the smooth background term $\boldsymbol{g}$.

\section{Discussion}

In this study we described a numerical method to restore energy dispersive X-ray diffraction data and to extract diffraction peaks. It is based on an inverse problem approach. It uses a linear direct model derived from an analysis of the formation of the data and the causes of diffraction peaks blurring. Using a continuous optimization technique, it then estimates the "perfect" diffraction spectrum that best reproduces the data according to the model and some additional priors. These priors are the sparsity of the crystalline diffraction spectrum and the smoothness of the baseline spectrum.

The level of influence of the priors are tuned using two hyper-parameters. In the present work the hyperparameters were set by trials and error for each spectrum. The setting of hyperparameters can be tedious and consequently it will be necessary to find an automatic method for routine use of this method. A further study will be necessary to determine whether existing hyper-parameters setting methods (L-curve[19], GCV[28], SURE[20]...) can be used or whether a specific method has to be defined.

This method was tested on real diffraction spectra of known crystalline materials: $\mathrm{NaCl}$ and graphite. Computation time were approximately equal to acquisition time (several minutes). On both materials, the presented method was able to extract precisely diffraction peaks even at high energy where the number of detected photons per channel is low $(<10)$. These real results clearly assess two facts: (i) the direct model is correct as it provides good fits of the data and (ii) the priors effectively provide satisfying solutions.

As attenuation and source spectra were only coarsely estimated, the estimated relative magnitude of the diffrac-

\section{Acknowledgment}

The algorithms presented in this article have been implemented in Yorick ${ }^{2}$, a free data processing language written by D. Munro. The authors are grateful to Éric Thiébaut whose fruitful advices and attentive reading have helped to improve this paper. This work has been supported by project SPIDERS funded by the French National Research Agency.

\section{Bibliography}

[1] J. Hanawalt, H. Rinn, Identification of crystalline materials. classification and use of x-ray diffraction patterns, Ind. Eng. Chem. Anal. Ed 8 (1936) 244-247.

[2] C. Crespy, P. Duvauchelle, V. Kaftandjian, F. Soulez, P. Ponard, Energy dispersive x-ray diffraction to identify explosive substances: Spectra analysis procedure optimization, $\mathrm{Nu}-$ clear Instruments and Methods in Physics Research Section A 623 (3) (2010) 1050 - 1060.

[3] J. Langford, D. Louër, Powder diffraction, Reports on Progress in Physics 59 (1996) 131.

[4] B. Buras, L. Gerward, X-Ray Energy Dispersive Diffraction, 1988.

[5] B. Buras, J. Olsen, L. Gerward, G. Will, E. Hinze, X-ray energydispersive diffractometry using synchrotron radiation, Journal of Applied Crystallography 10 (6) (1977) 431-438.

[6] A. Glazer, M. Hidaka, J. Bordas, Energy-dispersive powder profile refinement using synchrotron radiation, Journal of Applied Crystallography 11 (3) (1978) 165-172.

[7] A. Albinati, B. Willis, The rietveld method in neutron and x-ray powder diffraction, Journal of Applied Crystallography 15 (4) (1982) 361-374.

[8] Y. Dong, J. Liu, Y. Li, X. Li, A full-pattern fitting algorithm for energy-dispersive x-ray diffraction, Journal of Applied Crystallography 36 (5) (2003) 1123-1127.

[9] V. Honkimaki, P. Suortti, Energy-dispersive diffraction with synchrotron radiation and a germanium detector, Journal of Synchrotron Radiation 14 (4) (2007) 331-338.

\footnotetext{
${ }^{2}$ http://yorick.sourceforge.net
} 
[10] R. Luggar, J. Horrocks, R. Speller, R. Lacey, Determination of the geometric blurring of an energy dispersive x-ray diffraction (EDXRD) system and its use in the simulation of experimentally derived diffraction profiles, Nuclear Instruments and Methods in Physics Research Section A 383 (2-3) (1996) 610-618.

[11] A. Guinier, Theorie et Technique de la Radiocristallographie, 2nd Edition, Dunod, 1956.

[12] G. Harding, J. Kosanetzky, Status and outlook of coherent-xray scatter imaging, Journal of the Optical Society of America A 4 (5) (1987) 933-944.

[13] J. Delfs, J. Schlomka, Energy-dispersive coherent scatter computed tomography, Applied Physics Letters 88 (2006) 243506.

[14] S. Pani, E. Cook, J. Horrocks, L. George, S. Hardwick, R. Speller, Modelling an energy-dispersive x-ray diffraction system for drug detection, IEEE Trans. Nucl. Sci. 56 (3) (2009) 1238-1241.

[15] E. Thiébaut, J.-M. Conan, Strict a priori constraints for maximum likelihood blind deconvolution, J. Opt. Soc. Am. A 12 (3) (1995) 485-492.

[16] L. M. Mugnier, T. Fusco, J.-M. Conan, Mistral: a myopic edgepreserving image restoration method, with application to astronomical adaptive-optics-corrected long-exposure images, J. Opt. Soc. Am. A 21 (10) (2004) 1841-1854.

[17] D. Donoho, For most large underdetermined systems of equations, the minimal 11-norm near-solution approximates the sparsest near-solution, Communications on Pure and Applied Mathematics 59 (7) (2006) 907-934.

[18] S. Mallat, Z. Zhang, Matching pursuits with time-frequency dictionaries, IEEE Transactions on signal processing 41 (12) (1993) $3397-3415$.

[19] P. C. Hansen, Analysis of discrete ill-posed problems by means of the l-curve, SIAM Rev. 34 (4) (1992) 561-580.

[20] C. Stein, Estimation of the mean of a multivariate normal distribution, The Annals of Statistics 9 (6) (1981) 1135-1151.

[21] E. Thiébaut, Optimization issues in blind deconvolution algorithms, in: J.-L. Starck (Ed.), Astronomical Data Analysis II., Vol. 4847, 2002, pp. 174-183.

[22] J. Nocedal, S. Wright, Numerical optimization, Springer, 1999

[23] W. Bragg, The structure of some crystals as indicated by their diffraction of x-rays, Proceedings of the Royal Society of London. Series A, Containing Papers of a Mathematical and Physical Character 89 (610) (1913) 248-277.

[24] A. W. Hull, A new method of x-ray crystal analysis, Phys. Rev. 10 (6) (1917) 661-696

[25] R. Birch, M. Marshall, Computation of bremsstrahlung x-ray spectra and comparison with spectra measured with a ge (li) detector, Physics in Medicine and Biology 24 (1979) 505-517.

[26] H. Swanson, R. Fuyat, G. Ugrinic, Standard x-ray diffraction powder patterns, National Bureau of Standards Circ. 539 vol II (1953).

[27] M. Read, ASTM card 12-212, bell Telephone Laboratories, Murray Hill, New Jersey (1960).

[28] G. H. Golub, M. Heath, G. Wahba, Generalized cross-validation as a method for choosing a good ridge parameter, Technometrics 21 (1979) 215-223.

[29] F. Soulez, E. Thiébaut, Y. Tourneur, A. Gressard, R. Dauphin, Blind deconvolution of video sequences, in: Proceedings of 15th International Conférence on Image Processing, San Diego USA, 2008

[30] F. Soulez, C. Crespy, V. Kaftandjian, P. Duvauchelle, A. Peterzol, P. Ponard, Spatial super-resolution for line parallel imaging in energy dispersive $\mathrm{x}$-ray diffraction, in: European Conference on Non-Destructive Testing (ECNDT), 2010. 